\title{
Convection in protoneutron stars and the structure of surface magnetic fields in pulsars
}

\author{
V. Urpin ${ }^{1,2}$ and J. Gil ${ }^{3}$ \\ 1 A.F. Ioffe Institute of Physics and Technology and Isaak Newton Institute of Chili, Branch in St. Petersburg, \\ 194021 St. Petersburg, Russia \\ 2 Departament de Fisica Aplicada, Universitat d'Alacant, Ap. Correus 99, 03080 Alacant, Spain \\ 3 Institute of Astronomy, University of Zielona Gora, Lubuska 2, 65-265 Zielona Gora, Poland
}

Received 6 October 2003 / Accepted 3 November 2003

\begin{abstract}
We consider generation and evolution of small-scale magnetic fields in neutron stars. These fields can be generated by small-scale turbulent dynamo action soon after the collapse when the protoneutron star is subject to convective and neutron finger instabilities. After instabilities stop, small-scale fields should be frozen into the crust that forms initially at high density $\sim 10^{14} \mathrm{~g} / \mathrm{cm}^{3}$ and then spreads to the surface. Because of high crustal conductivity, magnetic fields with the lengthscale $\sim 1-3 \mathrm{~km}$ can survive in the crust as long as 10-100 Myr and form a sunspot-like structure at the surface of radiopulsars.
\end{abstract}

Key words. stars: pulsars: general - stars: neutron - magnetic fields

\section{Introduction}

Our knowledge of the magnetic field strength in neutron stars comes mainly from radiopulsars with measured spin-down rates. With the assumption that the spin-down torque on the pulsar is determined by its magnetodipole radiation (Ostriker $\&$ Gunn 1969), the spin period, $P$, and spin-down rate, $\dot{P}$, are related to the field strength at the magnetic pole, $B_{\mathrm{d}}$, by

$P \dot{P}=\frac{8 \pi^{2} B_{\mathrm{d}}^{2} R^{6}}{3 c^{2} I}$

where $I$ is the moment of inertia and $R$ is the radius (we assume that the magnetic and rotation axes are perpendicular). The magnetic fields inferred from the spin-down data range from $\sim 5 \times 10^{13}$ to $\sim 10^{8} \mathrm{G}$ but, most likely, these fields characterize the global magnetic configuration of neutron stars rather than a fine magnetic structure near the stellar surface.

Measurements of the spin-down rate are not, however, the only way to obtain information about the neutron star magnetic fields. Recent observations of the X-ray spectra features of some pulsars provide one more opportunity to look into the magnetic field near the surface. Absorbtion features in the spectrum of the isolated pulsar 1E 1207.4-5209 were associated by Sanwal et al. (2002) with atomic transitions of once-ionized helium in the neutron star atmosphere with a strong "surface" magnetic field, $B_{\mathrm{s}} \sim 1.5 \times 10^{14} \mathrm{G}$. An estimate of the spindown rate of this pulsar provides the strength of the "dipole" magnetic field, $B_{\mathrm{d}} \sim(2-4) \times 10^{12} \mathrm{G}$ (Pavlov et al. 2002) that is typical for radio pulsars of that age $(\sim 0.2-1.6 \mathrm{Myr})$.

Send offprint requests to: V. Urpin, e-mail: vadim. urpin@uv.es
Becker et al. (2002) found an emission line in the X-ray spectrum of PSR B1821-24 that could be interpreted as cyclotron emission from the corona above the pulsar's polar cap. This emission line is likely formed in a magnetic field $B_{\mathrm{s}} \sim$ $3 \times 10^{11} \mathrm{G}$ which is approximately two orders of magnitude stronger than the "dipole" field inferred from $P$ and $\dot{P}$. Haberl et al. (2003) interpreted a broad absorption feature in the spectrum of the isolated neutron star RBS1223 as a cyclotron line produced by protons in the magnetic field $\sim(2-6) \times 10^{13} \mathrm{G}$. These measurements provide strong evidence that the local magnetic fields on the neutron star surface can exceed the conventional "dipole" field.

Another piece of evidence on the distinction between the "dipole" and "surface" magnetic fields comes from the data on emission of radio pulsars. Recently, Gil \& Mitra (2001) and Gil \& Melikidze (2002) argued that the formation of a vacuum gap in radio pulsars is possible if the actual surface magnetic field near the polar cap is very strong, $B_{\mathrm{s}} \sim 10^{13} \mathrm{G}$, irrespective of the field measured from the $P-\dot{P}$ data. Also, radio emission from the recently discovered pulsar PSR J2144-3933 with the longest period $8.5 \mathrm{~s}$, which lies extremely far beyond the conventional death line, can be understood if this pulsar has a strong surface magnetic field of complex geometry (Gil \& Mitra 2001). Radio emission of many other pulsars which lie in the pulsar graveyard and should be radio silent can be explained if one adopts the model with a strong and complex surface field with a small curvature of the field lines $\left(<10^{6} \mathrm{~cm}\right)$. This model is consistent with the conclusion of Arons \& Scharlemann (1979) and Arons (1993) that pulsars with very long periods ( $\geq 5 \mathrm{~s}$ ) require a more complex field 
configuration than a dipole if pair creation is essential for the mechanism of radio emission. Analysing the phenomenon of drifting subpulses observed in many pulsars, Gil \& Sendyk (2000) found that their behaviour is consistent with the vacuum gap maintained by a strong sunspot-like magnetic field. Following this idea Gil et al. (2002b) found that in the famous case of PSR 0943+10 (Deshpande \& Rankin 1999, 2001) the "surface" field should be approximately 7 times stronger than the "dipole" component inferred from the spin-down rate. A sunspot-like configuration of the surface magnetic field is also suggested by the spin-down index in some pulsars (Cheng \& Ruderman 1993; Ruderman et al. 1998). Also, Cheng \& Zhang (1999), analysing the X-ray emission from the polar regions of the rotation-powered pulsars, argued that $B_{\mathrm{s}} \sim 10^{13} \mathrm{G}$ and the characteristic curvature of the field lines is around $10^{5} \mathrm{~cm}$ resembling the sunspot-like structure.

Rapidly growing amount of evidence on the distinction between the local field strength at the stellar surface and the global "dipole" field suggests that this can be a general phenomenon in neutron stars. In this paper, we propose a scenario of the formation of such complex magnetic configurations with the strength of a small scale "surface" field in excess of the "dipole" component. It is generally accepted that neutron stars are subject to hydrodynamic instabilities soon after their birth in the core collapse (see, e.g., Epstein 1979; Livio et al. 1980; Burrows \& Lattimer 1986). The convective stage lasts about 30-40 s (Miralles et al. 2000, 2002) and, under certain conditions, turbulent motions can amplify the magnetic field via dynamo action (Thompson \& Duncan 1993; Xu \& Busse 2001). The generated magnetic field must be frozen into the crust that is formed in the course of neutron star cooling. Because of high crustal conductivity, magnetic fields with a relatively small lengthscale, $\sim 10^{5} \mathrm{~cm}$, can survive during the active lifetime of radio pulsars.

The paper is organized as follows. In Sect. 2, we consider convection and associated dynamo action in protoneutron stars, and estimate the field that can be generated during this stage. We discuss the crust formation and small scale crustal magnetic structures in Sect. 3. Our results are briefly summarized in Sect. 4.

\section{Convection in protoneutron stars}

Neutron stars are formed in a supernova explosion associated to the gravitational collapse of massive stars. The explosion lasts $\sim 1 \mathrm{~s}$ and is followed by the core bounce and generation of a strong shock which heats the protoneutron star to a very high temperature $\sim 10^{11} \mathrm{~K}$ (Burrows \& Lattimer 1986; Burrows \& Fryxell 1992; Rampp \& Janka 2000). Hydrostatic equilibrium settles down on a very short timescale $\sim 10^{-3}-10^{-2} \mathrm{~s}$ but, even when the equilibrium is reached, the surface layers are very extended, thus the radius of a protostar is $\sim 50-100 \mathrm{~km}$ instead of the canonical 10-15 km. However, compression of the exterior zones is quite fast and, after a few seconds, the star is compressed to the canonical radius.

Hydrodynamic instabilities in a newly born neutron star are driven by both the lepton gradient (Epstein 1979), and the development of negative entropy gradients, which are common in many simulations of supernovae explosions (Bruenn \& Mezzacappa 1994, 1995; Rampp \& Janka 2000) and evolutionary models of protoneutron stars (Keil \& Janka 1995; Keil et al. 1996; Pons et al. 1999). Likely, both, the convective and neutron finger instabilities, can arise in protoneutron stars (Miralles et al. 2000) with the neutron finger unstable region typically surrounding the convective region. Initially, only the surface layer, containing around $0.5 M_{\odot}$, is unstable but the bottom of the unstable region spreads down to the center. Approximately in $10 \mathrm{~s}$, the whole star is subject to instability with convection, operating in the central region of the enclosed mass $\sim 0.5 M_{\odot}$, and the neutron finger instability dominating in the rest of the volume. After $\sim 20 \mathrm{~s}$, the unstable region begins to shrink to the center and, at $t \sim 40 \mathrm{~s}$, both the temperature and lepton gradients become too smooth to maintain instabilities. Both, the Rayleigh and Grashoff numbers are typically large in the unstable regions, and instabilities likely do operate in a turbulent regime (Thompson \& Duncan 1993).

During the hydrodynamic unstable phase, a protoneutron star is opaque to neutrino, and the turbulent velocity can be estimated by the standard mixing-length approximation (see, e.g., Schwarzschild 1958). The largest unstable lengthscale is of the order of the pressure lengthscale, $L$, and the turbulent velocity in this scale, $v_{L}$, can be estimated as

$v_{L} \approx \frac{L}{\tau_{L}}$

where $\tau_{L}$ is the instability growth time (which is of the order of the turnover time in a scale $L$ ). In the convectively unstable region, we have

$\frac{1}{\tau_{L}^{2}} \sim \frac{1}{\tau_{\mathrm{c}}^{2}}=\frac{1}{3} g \beta \frac{|\Delta \nabla T|}{T}$,

where $\tau_{\mathrm{c}}$ is the convection growth time, $g$ is the gravity, $\Delta \nabla T$ is the difference between the actual and adiabatic temperature gradients, and $\beta$ is the thermal expansion coefficient. The turbulent velocity, $v_{L}$, varies in time since $\Delta \nabla T$ progressively reduces due to the neutron star cooling. Convection is a dynamical instability and grows on a short timescale $(\sim 0.1-1 \mathrm{~ms}$ when convection is most efficient). Assuming $L \sim 1-3 \mathrm{~km}$ and using the calculations of $\tau_{\mathrm{c}}$ (Miralles et al. 2000), we can estimate $v_{L} \sim 10^{8} \mathrm{~cm} / \mathrm{s}$ (except the late unstable phase when convection is almost exhausted).

In the region of a "doubly diffusive" instability often referred to as "neutron fingers", a destabilizing influence of the lepton number gradient usually dominates the effect of $\Delta \nabla T$. This instability is the astrophysical analog of salt fingers that exist in terrestrial oceans. Physically, a fluid element perturbed downward in the protoneutron star can thermally equilibrate more rapidly with the background but find itself lepton-poorer and denser and, therefore, subject to a downward force that would amplify perturbations. This instability is typically more efficient in the region above the convective zone, involving a larger portion of the stellar material. With the same reasoning, we can estimate $v_{L}$ in this region substituting

$\frac{1}{\tau_{L}^{2}} \sim \frac{1}{\tau_{\mathrm{nf}}^{2}}=\frac{1}{3} g \delta|\nabla Y|$ 
into Eq. (2); $\tau_{\mathrm{nf}}$ is the neutron finger instability growth time, $\delta$ is the chemical expansion coefficient and $Y=\left(n_{\mathrm{e}}+n_{v}\right) / n$ is the lepton fraction with $n_{\mathrm{e}}, n_{v}$, and $n$ is the number density of electrons, neutrinos, and baryons, respectively. The neutron finger instability is typically slower than convection, $\tau_{\mathrm{nf}} \sim 30-100 \mathrm{~ms}$ (except the very early and very late phases). Being slower, this instability can nevertheless exist in a more extended region. Using the calculations of $\tau_{\mathrm{nf}}$ (Miralles et al. 2000), we estimate $v_{L} \sim(1-3) \times 10^{6} \mathrm{~cm} / \mathrm{s}$ in the neutron finger unstable region.

Likely, protoneutron stars rotate relatively rapidly (Zwerger \& Müller 1997; Rampp et al. 1998) and, hence, can be subject to the turbulent dynamo action. The initial spins of pulsars are not well constrained by observations but, most likely, they lie around $\sim 100 \mathrm{~ms}$ (Narayan 1987). The influence of rotation on turbulence is characterized by the Rossby number, $R o=P / \tau_{L}$ where $P$ is the spin period. Since there are two unstable regions inside the protoneutron star with substantially different properties, the Rossby number can differ much in the convective and neutron finger unstable regions. In the convective zone, we have $R o \sim 100$, and the influence of rotation on turbulence is probably negligible. On the contrary, in the neutron fingers unstable region, $R o \sim 1$ and turbulence can be strongly modified by the Coriolis force. Therefore, the neutron finger unstable region seems to be better suited for the mean-field dynamo action (see Bonanno et al. 2003). Note that this region is typically above the convectively unstable region, therefore the mean-field dynamo operates mainly in the surface layers.

In both unstable regions, however, turbulent motions can generate turbulent magnetic fields by small-scale dynamo action. The electrical conductivity of hot nuclear matter, $\sigma$, is relatively high, $\sigma \approx 1.5 \times 10^{24} T_{10}^{-2} \mathrm{~s}^{-1}$ where $T_{10}=T / 10^{10} \mathrm{~K}$ (Baym et al. 1971), and therefore the characteristic timescale of ohmic dissipation is very long $\left(\sim 10^{5}\right.$ yrs for the field with the lengthscale $L \sim 1-3 \mathrm{~km}$ ). The magnetic diffusivity, $\eta=$ $c^{2} / 4 \pi \sigma$, is small compared to viscosity, $v$, therefore the dynamo operates in the regime of large magnetic Prandtl numbers, $\operatorname{Pr}=v / \eta$. There are two factors that cause viscous stresses in protoneutron stars: neutrino transport and neutron scattering (Thompson \& Duncan 1993). Neutrino-induced viscosity dominates on scales larger than the neutrino mean-free path and can be very efficient. However, even this large viscosity cannot prevent instability on scales comparable to the pressure lengthscale (Miralles et al. 2000). The viscosity caused by neutron scattering operates on scales shorter than the neutrino mean-free path and is much smaller, but the magnetic Prandtl number is large even in this case,

$\operatorname{Pr}=\frac{v}{\eta} \approx 2.6 \times 10^{4} \rho_{14}^{5 / 4} T_{10}^{-4} \gg 1$,

where $\rho_{14}=\rho / 10^{14} \mathrm{~g} / \mathrm{cm}^{3}$ and $\rho$ is the density; we use the analytical fit for the coefficient of viscosity of hot nuclear matter obtained by Cutler et al. (1990).

There are two qualitatively different phases to a small-scale dynamo action: the kinematic phase when the field does not provide a noticeable influence on turbulent motions, and the dynamical phase when velocity is affected by the Lorentz force. In the kinematic regime, a weak seed magnetic field grows in strength exponentially in time, while the characteristic lengthscale of a field, $\ell_{B}$, decreases exponentially (Kazantsev 1968; Kraichnan 1976; Kulsrud \& Anderson 1992). In protoneutron stars, instabilities generate primary turbulent motions on scales $\sim L$, and these motions amplify turbulent magnetic fields of the same scale. However, the energy of such magnetic fluctuations is transfered to the small scales after a few eddy-turnover times. For $\operatorname{Pr} \gg 1$, the magnetic energy is first transfered to scales shorter than the viscous dissipative scale, $\ell_{v} \sim R e^{-3 / 4} L$ ( $R e$ is the Reynolds number at the lengthscale $L$ ), but greater than the magnetic dissipative lengthscale, $\ell_{\eta} \sim \ell_{v} \mathrm{Pr}^{-1 / 2}$ (see, e.g., Schekochihin et al. 2002). Both lengthscales, $\ell_{v}$ and $\ell_{\eta}$, are very small in protoneutron stars,

$\ell_{v} \approx 1.9 \times 10^{-5} v_{8}^{-3 / 4} L_{5}^{1 / 4} T_{10}^{-3 / 2} \rho_{14}^{15 / 16} \mathrm{~cm}$,

$\ell_{\eta} \approx 1.2 \times 10^{-7} v_{8}^{-3 / 4} L_{5}^{1 / 4} T_{10}^{1 / 2} \rho_{14}^{5 / 16} \mathrm{~cm}$,

where $v_{8}=v_{L} / 10^{8} \mathrm{~cm} / \mathrm{s}$, and $L_{5}=L / 10^{5} \mathrm{~cm}$. Turbulent motions with the lengthscale shorter than $\ell_{v}$ are suppressed by viscosity, but magnetic fluctuations can exist if their lengthscale is larger than $\ell_{\eta}$. Probably, turbulence is well developed in both unstable zones, and fluctuations spread through a wide range of scales (see, e.g., Thompson \& Duncan 1993). The kinematic growth phase is terminated when the Lorentz force starts modifying the convective motions. Since the characteristic growth time of small-scale fields, $\sim L / v_{L}$, is much shorter than the duration of the unstable phase, the small-scale dynamo operates likely in a nonlinear regime in both unstable regions.

In the non-linear regime, turbulence drives kinetic and magnetic energy cascades which are quasi-steady in protoneutron stars. The nature of these cascades in MHD turbulence has been a matter of debate for many years. Iroshnikov (1963) and Kraichnan (1965) suggested that in the inertial range kinetic and magnetic power spectra are given by

$E_{\mathrm{K}}(\ell) \propto E_{\mathrm{M}}(\ell) \propto \ell^{3 / 2}$,

where $\ell$ is the lengthscale of fluctuations. On the other hand, solar wind data suggest a spectrum $\propto \ell^{5 / 3}$ as in the Kolmogorov theory. Numerical simulations (see, e.g., Kida et al. 1991; Haugen et al. 2003) indicate that the magnetic energy spectrum probably does not show a power law behaviour. It seems that spectra can be even shallower than $\ell^{3 / 2}$ in some models. For any more or less plausible spectra, however, the amplitude of magnetic fluctuations increases with $\ell$ and reaches its maximum on scales comparable to the main lengthscale of turbulence, $L$. In a saturated phase, small-scale magnetic fields are approximately in equipartition with velocity fluctuations,

$B_{\ell} \sim B_{e q}(\ell)=\sqrt{4 \pi \rho} v_{\ell}$,

where $B_{\ell}$ and $v_{\ell}$ are the amplitudes of magnetic and velocity fluctuations with the lengthscale $\ell$. Equation (9) yields only the order of magnitude estimate, since the magnetic and kinetic energy have generally different spectra (Kida et al. 1991).

Turbulence is non-stationary in both unstable zones of protoneutron stars. It arises very rapidly soon after the collapse, reaches some quasi-steady regime, and then goes down 
when the temperature and lepton gradients are smoothed (after $\sim 30-40 \mathrm{~s}$ ). The timescale required for fluid to make one turn in a turbulent cell with the lengthscale $\ell$ can be estimated as $\tau_{\ell} \approx \pi \ell / v_{\ell}$. This timescale varies with time, but is typically much shorter than the characteristic cooling timescale, $\tau_{\text {cool }}$, except the very late phase when gradients are smoothed and instabilities are less efficient. Therefore, turbulence can be treated in a quasi-steady approximation during the almost whole unstable phase. Using Eq. (9), we can estimate the maximum field generated in the lengthscale $\ell \sim L$ during the quasisteady regime as $B_{L c} \sim 10^{16} \mathrm{G}$ in the convective zone, and $B_{L n f} \sim(1-3) \times 10^{14} \mathrm{G}$ in the neutron finger unstable zone. However, the temperature and lepton number gradients are progressively reduced as the protoneutron star cools down and, therefore, the turbulent velocity decreases as well. As the result, the strength of small-scale magnetic fields generated by turbulence also decreases compared to the maximum value, but estimate (9) is still valid until the quasi-steady condition $\tau_{\text {cool }} \gg \tau_{\ell}$, is fulfilled. We assume that this condition breaks down at $t=t_{a}$ when $\tau_{\ell}(t)$ becomes comparable to the cooling timescale: $\tau_{\ell}\left(t_{a}\right) \sim \tau_{\text {cool }}\left(t_{a}\right)$. Then, the turbulent velocity at $t=t_{a}$ is given by

$v_{\ell}\left(t_{a}\right) \sim \frac{\pi \ell}{\tau_{\mathrm{cool}}\left(t_{a}\right)}$

We assume that the final strength of the magnetic field generated by the small-scale dynamo, $B_{\ell}$, is of the order of $B_{\text {eq }}(\ell)$ at $t=t_{a}$. Perhaps this estimate provides only the upper limit on a field strength since turbulent motions can influence magnetic fluctuations even at $t>t_{a}$. However, the decay of turbulence is rather fast, and the kinetic energy of turbulence rapidly becomes smaller than the magnetic energy. Therefore, we have for the final strength of the generated magnetic field

$B_{\ell} \sim q \sqrt{4 \pi \rho} v_{\ell}\left(t_{a}\right)$,

where $q$ is a numerical factor, $q \sim 1$. Substituting expression (10) for $v_{\ell}\left(t_{a}\right)$, we obtain

$B_{\ell} \sim \frac{q \pi \sqrt{4 \pi \rho} \ell}{\tau_{\mathrm{cool}}\left(t_{a}\right)}$.

The final strength of the generated small-scale field turns out to be the same for both unstable zones and decreases with decreasing lengthscale. For the largest turbulent scale, $\ell=L \sim$ $1-3 \mathrm{~km}$, estimate (12) yields $B_{L} \sim 3 \times 10^{13} q \mathrm{G}$ if $\tau_{\text {cool }}$ is of the order of a few seconds. Note that our estimate is in contrast to that of Thompson \& Duncan (1993) who assumed that the strength of the generated field is approximately given by its maximum value.

\section{Crust formation and crustal small-scale magnetic fields}

It is difficult to predict final disposition of the magnetic field after the turbulent motions inside the protoneutron star stop. Most likely, there exists a wide spectrum of magnetic fluctuations with the lengthscale shorter than $L$ and with the field strength given by Eq. (12). When convection is exhausted, these smallscale fluctuations evolve under the influence of the ohmic dissipation and buoyancy. After a magnetohydrodynamic quasiequilibrium is established, one expects that magnetic loops densely fill the volume and surface of the star, although the field strength may vary considerably, depending on the degree of intermittency of the generated small-scale field. In liquid nuclear matter, the ohmic decay timescale of fluctuations with the lengthscale $\ell$ is

$\tau_{\mathrm{O}}(\ell) \sim \frac{4 \pi \sigma \ell^{2}}{c^{2}}=2.8 \times 10^{7} T_{10}^{-2}\left(\frac{\ell}{L}\right)^{2} \mathrm{yrs}$,

and dissipation is important only for fluctuations with very small $\ell$.

Likely, the crust formation provides the most important influence on the evolution of small-scale magnetic fields at this stage. Approximately at the age $440-50 \mathrm{~s}$ (soon after convection stops), the neutron star cools down to the internal temperature $\sim(1-3) \times 10^{10} \mathrm{~K}$ (Pons et al. 1999). At such temperature, neutrons and protons can form nuclei and clusters in the nuclear matter with the density $\sim 10^{14} \mathrm{~g} / \mathrm{cm}^{3}$. When the neutron star cools down to a lower temperature, nuclei can be formed at $\rho<10^{14} \mathrm{~g} / \mathrm{cm}^{3}$ as well. Note that nuclear composition at high density depends generally on the pre-history of the neutron star. For instance, the composition of "ground state" matter (Negele \& Vautherin 1973) differs noticeably from that of "accreted" matter (Haensel \& Zdunik 1990). The Coulomb interaction of nuclei leads to the crystal formation even at a relatively high temperature. Crystallization occurs when the ion coupling parameter $\Gamma=Z^{2} e^{2} /\left(a k_{\mathrm{B}} T\right)$ reaches the critical value $\Gamma=\Gamma_{\mathrm{m}} \approx 170$ (Slattery et al. 1980); $a=\left(3 / 4 \pi n_{i}\right)^{1 / 3}$ is the mean inter-ion distance, $n_{i}$ and $Z$ are the number density and charge number of ions, respectively; $T$ is the temperature, and $k_{\mathrm{B}}$ is the Boltzmann constant. Then, the crystallization temperature is

$T_{\mathrm{m}}=\frac{Z^{2} e^{2}}{a k_{\mathrm{B}} \Gamma_{\mathrm{m}}} \approx 1.3 \times 10^{7} Z^{5 / 3} \mu_{\mathrm{e}}^{-1 / 3} \rho_{12}^{1 / 3} \frac{170}{\Gamma_{\mathrm{m}}} \mathrm{K}$,

where $\mu_{\mathrm{e}}$ is the number of baryons per one electron, and $\rho_{12}=\rho / 10^{12} \mathrm{~g} / \mathrm{cm}^{3}$. For the density $\rho \sim 10^{13}-10^{14} \mathrm{~g} / \mathrm{cm}^{3}$, the crystallization temperature is of the order of $10^{10} \mathrm{~K}$ (Baiko \& Yakovlev 1996). Therefore, the crust formation starts almost immediately after the end of the convective phase, and the magnetic fields generated by convective motions should be frozen into the crust. Solidification proceeds rather rapidly, and the outer boundary of the crust reaches the density $\sim 10^{10} \mathrm{~g} / \mathrm{cm}^{3}$ after $\sim 1$ day in the case of standard cooling.

In crystal layers, the magnetic field evolution is mainly determined by ohmic dissipation. The crust electric conductivity $\sigma_{\mathrm{c}}$, can be expressed in terms of the electron relaxation time, $\tau_{\mathrm{e}}$ (see, e.g., Baiko \& Yakovlev 1996),

$\sigma_{\mathrm{c}} \approx 1.5 \times 10^{22} x^{2} \beta\left(\frac{\tau_{\mathrm{e}}}{10^{-16} \mathrm{~s}}\right) \mathrm{s}^{-1}$,

where $x=p_{\mathrm{F}} / m_{\mathrm{e}} c, \beta=v_{\mathrm{F}} / c ; p_{\mathrm{F}}$ and $v_{\mathrm{F}}$ are the electron Fermi momentum and velocity, respectively. In a high density region, electrons are ultrarelativistic and $\beta \approx 1$. Using expression (15), 
we can estimate the ohmic decay timescale of magnetic fluctuations with the lengthscale $\ell$ in the crust,

$\tau_{\mathrm{Oc}}(\ell)=\frac{4 \pi \sigma_{\mathrm{c}} \ell^{2}}{c^{2}} \approx 0.7 \times 10^{5} x^{2} \beta\left(\frac{\tau_{\mathrm{e}}}{10^{-16} \mathrm{~s}}\right) \ell_{5}^{2} \mathrm{yrs}$,

where $\ell_{5}=\ell / 10^{5} \mathrm{~cm}$. This timescale is short for short lengthscale fluctuations, but $\tau_{\mathrm{Oc}}$ can be much longer than the cooling timescale for fluctuations with relatively large $\ell$. For instance, the decay of fluctuations with $\ell \sim 10^{3}-10^{5} \mathrm{~cm}$ proceeds certainly on a timescale longer than the time required for the formation of a well developed crust. Therefore, turbulent magnetic fields with relatively large lengthscales are likely frozen into the crystallized matter soon after convection stops.

Further evolution of turbulent magnetic fields deposed in the neutron star crust depends on conductive properties of the crustal matter and the cooling scenario. The behaviour of such fields is governed by the standard induction equation where only the ohmic dissipation is included and is qualitatively similar to the behaviour of a large scale crustal magnetic field considered in detail by Urpin \& Konenkov (1997). We refer the results of this paper in what follows. We consider the evolution of turbulent fields for the neutron star model with the standard cooling since this cooling scenario can better account for the available observational data. Note that models with accelerated cooling always lead to a slower decay of the magnetic field (Urpin \& van Riper 1993) and, as a result the small-scale magnetic structure can survive for a longer time in such models. The crustal conductivity is determined by scattering of electrons on phonons and impurities. Scattering on phonons dominates the conductivity while the neutron star is relatively hot whereas impurities give the main contribution at a low crustal temperature (see, e.g., Yakovlev \& Urpin 1980). The effect of impurities on the conductivity is characterized by the impurity parameter $Q$,

$Q=\frac{1}{n} \sum_{n^{\prime}} n^{\prime}\left(Z-Z^{\prime}\right)^{2}$,

where $n^{\prime}$ is the number density of an interloperspecies of charge $Z^{\prime}, n$ and $Z$ are the number density and charge of the dominant ion species; summation is over all species. Most likely, $Q$ ranges from 0.001 to 0.1 within the crust.

In Fig. 1, we plot the time dependence of the surface strength of a small-scale magnetic field for a neutron star model with mass $M=1.4 M_{\odot}$ and with the equation of state of Pandharipande and Smith (see, e.g., Pandharipande et al. 1976). The radius of this model is $R=15.98 \mathrm{~km}$. For the sake of simplicity, we assume in calculations that the dependence of a field on polar and azimuthal coordinates is sinusoidal with the wavelength equal to the main scale of turbulence, $L$, and use the so called local approximation in these two directions. This is a sufficiently good approximation since the radius of the star is substantially larger than $L$ and the thickness of the crust. We also assume that the initial radial depth of a small-scale field is equal to the main scale of turbulence, $L$, and varies within the range $1-2 \mathrm{~km}$. This range of depth corresponds to the density from $\approx 5 \times 10^{12} \mathrm{~g} / \mathrm{cm}^{3}$ to $\approx 10^{14} \mathrm{~g} / \mathrm{cm}^{3}$ for the PS-model. The initial radial dependence of the field is chosen in accordance

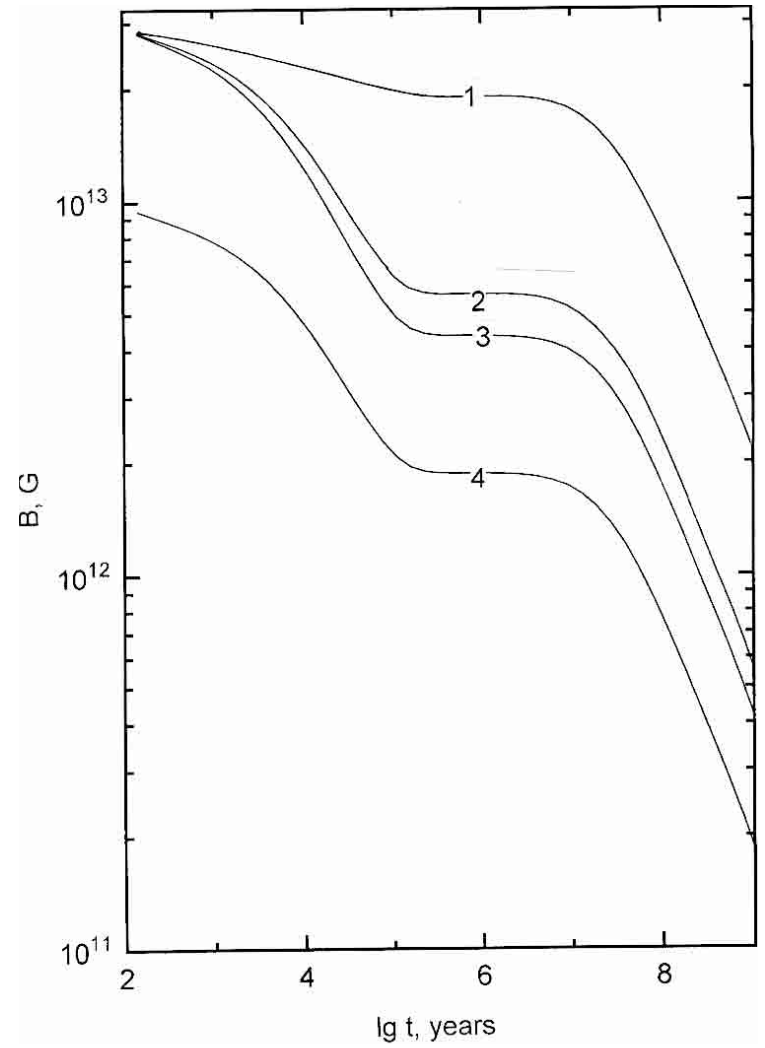

Fig. 1. The evolution of the surface magnetic field strength $B$ for different initial lengthscales $L$. The decay curves are shown for $L=2 \mathrm{~km}$ (curve 1), $1.2 \mathrm{~km}$ (curves 2 and 4 ), and $1.0 \mathrm{~km}$ (curve 3 ). The initial field is $3 \times 10^{13} \mathrm{G}$ for the curves 1,2 and 3 , and $10^{13} \mathrm{G}$ for the curve 4 . The crustal impurity parameter is $Q=0.01$.

with the model proposed by Urpin \& Konenkov (1997). Note that main conclusions of our paper are not sensitive to a particular choice of this dependence but are flexible to the value of $L$.

The magnetic field evolution is shown for three initial depths of the field, $L=1 \mathrm{~km}$ (this depth corresponds to the density $\rho \approx 5 \times 10^{12} \mathrm{~g} / \mathrm{cm}^{3}$ in the crust), $L=1.2 \mathrm{~km}$ $\left(\rho \approx 10^{13} \mathrm{~g} / \mathrm{cm}^{3}\right)$, and $L=2 \mathrm{~km}\left(\rho \approx 10^{14} \mathrm{~km}\right)$. For the purpose of illustration, the decay in the case $L=1.2 \mathrm{~km}$ is shown for two initial field strength, $3 \times 10^{13} \mathrm{G}$ and $10^{13} \mathrm{G}$ (curves 2 and 4 , respectively). During the initial stage ( $t \leq 10^{5} \mathrm{yrs}$ ), the crustal conductivity is determined by scattering of electrons on phonons and is relatively low for all calculated models. Therefore, the field decay can be essential during this stage, and the surface field strength can weaken by a factor $\approx 2-7$, depending on the lengthscale of the initial turbulent field. Obviously, a decrease during the initiale stage is smaller for the field with larger $L$. For instance, a small-scale field with $L=1 \mathrm{~km}$ is reduced by a factor $\approx 7$ after $10^{5}$ yrs whereas the field with $L=2$ $\mathrm{km}$ decreases less than twice after the same time. Note that for $L>2 \mathrm{~km}$ the decrease during the initial stage is practically negligible, and the field at $t \sim 10^{5} \mathrm{yrs}$ is only a bit lower than the initial field.

After $\sim 10^{5} \mathrm{yrs}$, the dominant conductivity mechanism changes from electron-phonon to electron-impurity scattering. As a result, the conductivity increases and the rate of field 
decay slows down. We calculate the field decay during this stage using an intermediate value of the impurity parameter, $Q=0.01$ that characterizes not a very poluted crust. For larger $Q$, the decay is more rapid (see, for comparison, Urpin \& Konenkov 1997). The evolution of small-scale fields shows the presence of flat segments of the decay curves at $t \sim 0.1-100$ Myr like those of a large-scale field. The length of a plateau depends on the impurity parameter, $Q$. The lower that $Q$ gets, the longer the plateau on the corresponding decay curve is. During the impurity dominating stage, the decay turns out to be extremely slow, and the characteristic decay time can be as long as 10-100 Myr.

These simple model calculations show very clearly that turbulent magnetic fields with the lengthscale of the order of the turbulence main lenghtscale in protoneutron stars $(\sim 1-3 \mathrm{~km})$ can survive in the crust during a very long time $\sim 10-100 \mathrm{Myr}$ that is generally comparable to the active lifetime of radiopulsars. The field strength in such magnetic spots on the surface can reach $5 \times 10^{12}-2 \times 10^{13} \mathrm{G}$ depending on the radius of a spot and can be larger than (or comparable to) the strength of the dipole field.

\section{Conclusion}

We have considered the formation and evolution of small-scale magnetic structures in the neutron star surface layers. A wide spectrum of these structures can be generated by the smallscale turbulent dynamo action during the unstable phase that lasts $\sim 30-40 \mathrm{~s}$ after the neutron star birth. There are two substantially different unstable regions in the protoneutron star, with the convective instability active in the inner region and the neutron-finger instability more efficient in the outer region. Generally, the small-scale dynamo can generate smallscale magnetic structures in both unstable zones. Due to high conductivity of nuclear matter, the lengthscale of generated magnetic structures spreads from the main scale of turbulence, $L \sim 1-3 \mathrm{~km}$ (comparable to the pressure scale height), to extremely short lengthscales determined by ohmic dissipation. After instabilities stop, structures with short lengthscales decay on a short timescale $\propto \ell^{2}$ due to finite electrical conductivity whereas fields with larger lengthscales can survive for a longer time. The crust formation that starts almost immediately after instabilities stop may have the decisive influence on the evolution of such magnetic fields. These magnetic structures can be frozen into the crystallized matter and then evolve in the crustal layers. The neutron star cooling increases the conductivity of the crust and, as a result, small-scale magnetic structures decay extremely slowly. Our calculations show that structures with the lengthscale $L \sim 1-3 \mathrm{~km}$ can survive as long as $10-100 \mathrm{Myr}$ that is basically comparable to the active life-time of radiopulsars.

The strength of the magnetic field generated by the smallscale dynamo action is approximately determined by equipartition and decreases with the decreasing lengthscale. For magnetic structures with $L \sim 1-3 \mathrm{~km}$, the magnetic field can be as strong as $\sim 5 \times 10^{12}-2 \times 10^{13} \mathrm{G}$ even for radiopulsars as old as $\sim 10-100$ Myr. Note that the decay of small-scale magnetic fields is qualitatively similar to that of the large-scale crustal field considered by Urpin \& Konenkov (1997). The origin of a large-scale field in neutron stars is still debatable, but it is possible that this field was generated by some mechanism in the layer with $\rho<2 \times 10^{14} \mathrm{~g} / \mathrm{cm}^{3}$ that corresponds to the crust (see, e.g., Bonanno et al. 2003). Then, the thickness of a layer occupied by the large-scale field can generally differ from $L$. If the initial depth of a large-scale field is smaller than $L$, then the small-scale field decreases slower, and we observe a radiopulsar with magnetic "spots" where the field is stronger than the dipole field inferred from the spin-down rate. On the contrary, if the initial depth of a large-scale field is larger than $L$, then small-scale structures decay faster than the dipole field, and the resulting magnetic field becomes more regular with the age.

Small-scale magnetic structures with $L \sim 1-3 \mathrm{~km}$ and $B \sim 5 \times 10^{12}-2 \times 10^{13} \mathrm{G}$ at the surface may have an important influence on many properties of radiopulsars. As already mentioned in the Introduction, this range of the surface magnetic field is favourable for the inner vacuum gap formation in pulsars (Gil \& Melikidze 2002). The sparking discharge of this gap produces filaments of electron-positron plasma, whose presence seems absolutely necessary for generation of coherent pulsar radio emission. Moreover, the $\boldsymbol{E} \times \boldsymbol{B}$ drift of spark plasma should be manifested as the observed subpulse drift, provided that the actual surface field has some degree of axial symmetry, with a tendency to converge at the local pole (see Gil et al. 2003 for review). These authors assumed that one small-scale structure (a "spot") coincides to some extent with the canonical (dipolar) polar cap in the sence that the magnetic field lines form a complex magnetic configuration near the pole but connect smoothly with a subset of open dipolar field lines at a larger radius (Gil et al. 2002a). This means that the actual polar cap is defined by those non-dipolar field lines which penetrate the light cylinder.

A study of small-scale magnetic structures can also provide information regarding the very early evolutionary stage of neutron stars since the observed fields were frozen into the crust soon after the neutron star birth. For example, the presence of small-scale magnetic structures in radiopulsars can be a good evidence that protoneutron stars pass the turbulent stage in their evolution.

Acknowledgements. One of the authors (V.U.) thanks the University of Alicante for hospitality and the Spanish Ministry of Science and Technology for a financial support (grant AYA20013490-C02-02). This paper is supported in parts by the Polish KBN Grant 2 P03D 00819 and the grant 04-02-16243 of the Russian Foundation of Basic Research. J.G. acknowledges the renewal of the Alexander von Humboldt fellowship.

\section{References}

Arons, J. 1993, ApJ, 408, 160

Arons, J., \& Scharlemann, E. 1979, ApJ, 231, 854

Baiko, D., \& Yakovlev, D. 1996, Astr. Lett., 22, 708

Baym, G., Pethick, C., \& Sutherland, P. 1971, ApJ, 170, 299

Becker, W., Swartz, D., Pavlov, G., et al. 2003, ApJ, 594, 798

Bonanno, A., Rezzolla, L., \& Urpin, V. 2003, A\&A, 410, L33

Bruenn, S., \& Mezzacappa, A. 1994, ApJ, 433, L45

Bruenn, S., \& Mezzacappa, A. 1995, Phys. Rev., 256, 69 
Burrows, A., \& Fryxell, B. A. 1992, Science, 258, 430

Burrows, A., \& Lattimer, J. 1986, ApJ, 307, 178

Cheng, K., \& Ruderman, M. 1993, ApJ, 402, 264

Cutler, C., Lindblom, L., \& Splinter, R. 1990, ApJ, 230, 847

Deshpande, A. A., \& Rankin, J. M. 1999, ApJ, 524, 1008

Deshpande, A. A., \& Rankin, J. M. 2001, MNRAS, 322, 438

Epstein, R. 1979, MNRAS, 188, 305

Gil, J., \& Melikidze, G. 2002, ApJ, 577, 909

Gil, J., Melikidze, G., \& Geppert, U. 2003, A\&A, 407, 315

Gil, J., Melikidze, G., \& Mitra, D. 2002a, A\&A, 388, 235

Gil, J., Melikidze, G., \& Mitra, D. 2002b, A\&A, 388, 246

Gil, J., \& Mitra, D. 2001, ApJ, 550, 383

Gil, J., \& Sendyk, M. 2000, ApJ, 541, 351

Haberl, F., Schwope, A., Hambaryan, V., Hasinger, G., \& Motch, C. 2003, A\&A, 403, 19L

Haensel, P., \& Zdunik, J. 1990, A\&A, 227, 431

Haugen, N., Brandenburg, A., \& Dobler, W. 2003, ApJ, 597, L141

Iroshnikov, R. S. 1963, Sov. Astron., 7, 566

Kazantsev, A. 1968, Soviet Phys. JETP, 26, 1031

Keil, W., \& Janka, H.-T. 1995, A\&A, 296, 145

Keil, W., Janka, H.-T., \& Müllerr, E. 1996, ApJ, 473, L111

Kida, S., Yanase, S., \& Mizushima, J. 1991, Phys. Fluids, A3, 457

Kraichnan, R. H. 1965, Phys. Fluids, 8, 1385

Kraichnan, R. H. 1976, J. Fluid Mech., 75, 657

Kulsrud, R. M., \& Anderson, S. W. 1992, ApJ, 396, 606
Livio, M., Buchler, J., \& Colgate, S. 1980, ApJ, 238, L139

Miralles, J., Pons, J., \& Urpin, V. 2000, ApJ, 543, 1001

Miralles, J., Pons, J., \& Urpin, V. 2002, ApJ, 574, 356

Narayan, R. 1987, ApJ, 319, 162

Negele, J., \& Vautherin, D. 1973, Nucl. Phys., A207, 298

Ostriker, J. P., \& Gunn, J. E. 1969, ApJ, 157, 1395

Pandharipande, V. R., Pines, D., \& Smith, R. 1976, ApJ, 208, 550

Pavlov, G., Zavlin, V., Sanwal, D., \& Trümper, J. 2002, ApJ, 569, L95

Pons, J., Reddy, S., Prakash, M., Lattimer, J., \& Miralles, J. 1999, ApJ, 513, 780

Rampp, M., \& Janka, H.-T. 2000, ApJ, 539, L33

Rampp, M., Müller, E., \& Ruffert, M. 1998, A\&A, 332, 969

Ruderman, M., Zhu, T., \& Cheng, K. 1998, ApJ, 492, 267

Sanwal, D., Pavlov, G., Zavlin, V., \& Teter, M. 2002, ApJ, 574, L61

Schekochihin, A., Boldyrev, S., \& Kulsrud, R. 2002, ApJ, 567, 828

Schwarzschild, M. 1958, Structure and Evolution of the Stars (Princeton: Princeton Univ. Press)

Slattery, V., Doolen, G., \& De Witt, H. 1980, Phys. Rev. A, 21, 2087

Thompson, C., \& Duncan, R. 1993, ApJ, 408, 194

Urpin, V., \& Konenkov, D. 1997, MNRAS, 292, 167

Urpin, V., \& van Riper, K. 1993, ApJ, 411, L87

Xu, R. X., \& Busse, F. H. 2001, A\&A, 371, 963

Yakovlev, D., \& Urpin, V. 1980, SvA, 24, 303

Zwerger, T., \& Müller, E. 1997, A\&A, 320, 209 